\title{
Outcomes Following SARS-CoV-2 Infection in Patients With Primary and Secondary Immunodeficiency in The United Kingdom
}

Adrian M Shields

University of Birmingham https://orcid.org/0000-0001-5345-2156

Ariharan Anantharachagan

Lancashire Teaching Hospitals NHS Foundation Trust

Gururaj Arumugakani

St James University Hospital

Kenneth Baker

Newcastle University and Newcastle upon Tyne Hospitals NHS Foundation Trust

\section{Sameer Bahal}

Royal Free London NHS Foundation Trust

Helen Baxendale

Royal Papworth NHS Foundation Trust

William Bermingham

University Hospitals Birmingham NHS Foundation Trust

Malini Bhole

The Dudley Group NHS Foundation Trust

Evon Boules

Sheffield Teaching Hospitals NHS Foundation Trust

Philip Bright

Clinical Immunology, North Bristol NHS Trust

Charu Chopra

Department of Haematology \& Immunology, Royal Infirmary of Edinburgh

Lucy Cliffe

Nottingham University Hospital NHS Trust

Betsy Cleave

Nottingham University Hospital NHS Trust

John Dempster

University College London Hospitals

Lisa Devlin

Regional Immunology Service, The Royal Hospitals

Fatima Dhalla

Oxford University Hospitals NHS Foundation Trust

Lavanya Diwakar

Royal Stoke Hospital, Stoke-on-Trent

Elizabeth Drewe

Immunity and Inflammation Theme, Newcastle University

Christopher Duncan

University College London

\section{Magdalena Dziadzio}

Royal Victoria Infirmary, Newcastle upon Tyne Hospitals NHS Foundation Trust

\section{Suzanne Elcombe}

Salford Royal NHS Foundation Trust, Manchester

Shuayb Elkhalifa

ewcastle University, and Paediatric Stem Cell Transplant Unit, Great North Children's Hospital

Andrew Gennery

University Hospital Southampton NHS Trust

Harichandrana Ghanta

Royal London Hospital, Barts Health NHS Trust

Sarah Goddard

Department of Immunology, Royal Stoke Hospital

Sofia Grigoriadou 
Immunology Department, Royal London Hospital, Barts Health NHS Trust

\section{Scott Hackett}

University Hospitals of Birmingham

Grant Hayman

Epsom and St Helier University Hospital NHS Trust

Richard Herriot

Immunology Department, Aberdeen Royal Infirmary

\section{Archana Herwadkar}

Immunology Department, Salford Royal NHS Foundation Trust

\section{Aarnoud Huissoon}

University Hospitals Birmingham NHS Foundation Trust

Rashmi Jain

Oxford University Hospitals NHS Foundation Trust

\section{Stephen Jolles}

University Hospital of Wales

\section{Sarah Johnston}

Clinical Immunology, North Bristol NHS Trust

\section{Sujoy Khan}

Hull University Teaching Hospitals NHS Trust

James Laffan

Epsom and St Helier University Hospital NHS Trust

\section{Peter Lane}

University of Birmingham

Lucy Leeman

University Hospitals Plymouth NHS Trust

David M. Lowe

Institute of Immunity and Transplantation, University College London

\section{Shanti Mahabir}

Clinical Immunology and Allergy Department, Leicester Royal Infirmary

\section{Dylan James Mac Lochlainn}

Oxford University Hospitals NHS Foundation Trust

\section{Elizabeth McDermott}

Nottingham University Hospital NHS Trust

Siraj Misbah

Oxford University Hospitals NHS Foundation Trust

\section{Fiona Moghaddas}

Clinical Immunology, North Bristol NHS Trust

Hadeil Morsi

Oxford University Hospitals NHS Foundation Trust

\section{Sai Mumg}

Epsom and St Helier University Hospital NHS Trust

Sadia Noorani

Sandwell \& West Birmingham Hospitals NHS Trust

Rachael O’Brien

Department of Clinical Immunology, Frimley Park Hospital

Smita Patel

Oxford University Hospitals NHS Foundation Trust

Arthur Price

Clinical Immunology and Allergy Department, Leicester Royal Infirmary

\section{Tasneem Rahman}

Epsom and St Helier University Hospital NHS Trust

Suranjith Senevirantne

University College London

\section{Anna Shrimpton}

Sheffield Teaching Hospitals NHS Foundation Trust

Catherine Stroud

Royal Victoria Infirmary, Newcastle upon Tyne Hospitals NHS Foundation Trust

\section{Moira Thomas}

Clinical Immunology Service, NHS Greater Glasgow and Clyde 
Katie Townsend

Epsom and St Helier University Hospital NHS Trust

Prashantha Vaitla

Nottingham University Hospital NHS Trust

Nisha Verma

University College London

Anthony Williams

University Hospital Southampton NHS Trust

Siobhan O. Burns

University College London

Sinisa Savic

St James University Hospital

Alex G. Richter ( $\square$ a.g.richter@bham.ac.uk)

University of Birmingham

\section{Research Article}

Keywords: SARS-CoV-2, infection, immunodeficiency, COVID-19

Posted Date: October 8th, 2021

DOI: https://doi.org/10.21203/rs.3.rs-920110/v1

License: (c) (1) This work is licensed under a Creative Commons Attribution 4.0 International License. Read Full License

Version of Record: A version of this preprint was published at Clinical \& Experimental Immunology on January 31 st, 2022. See the published version at https://doi.org/10.1093/cei/uxac008. 


\section{Abstract \\ Purpose}

To define the burden of morbidity and mortality arising from COVID-19 in individuals with primary (PID) and secondary immunodeficiency (SID) in the United Kingdom.

\section{Methods}

In March 2020, the United Kingdom Primary Immunodeficiency Network (UKPIN) established a registry of cases to collate the outcomes of individuals with PID and SID following SARS-CoV-2 infection and treatment. Anonymised demographic data, pre-SARS-CoV-2 infection lymphocyte counts, co-morbidities, targeted treatments and outcomes were collected. Three groups were analysed in further detail: individuals with common variable immunodeficiency (CVID), individuals with any PID, including CVID, receiving immunoglobulin replacement therapy (IgRT) and individuals with secondary immunodeficiency.

\section{Results}

A total of 310 cases of SARS-CoV-2 infection in individuals with PID or SID have now been reported in the UK. The overall mortality within the cohort was $17.7 \%(n=55 / 310)$. Individuals with CVID demonstrated an infection fatality rate (IFR) of $18.3 \%(n=17 / 93)$, individuals with PID receiving IgRT had an IFR of $16.3 \%(n=26 / 159)$ and individuals with SID, an IFR of $27.2 \%(n=25 / 92)$. Individuals with PID and SID, had higher inpatient mortality and died at a younger age than the general population. Increasing age, low pre-SARS-CoV-2 infection lymphocyte count and the presence of common co-morbidities increased the risk of mortality in PID. Access to specific COVID-19 treatments in this cohort was limited: only $22.9 \%(n=33 / 144)$ of patients admitted to hospital received dexamethasone, remdesivir, an anti-SARS-CoV-2 antibody-based therapeutic (e.g. REGN-COV2 or convalescent plasma) or tocilizumab as a monotherapy or in combination. Dexamethasone, remdesivir and anti-SARS-CoV-2 antibody-based therapeutics appeared efficacious in PID and SID.

\section{Conclusion}

Compared to the general population, individuals with PID or SID are at high risk of mortality following SARS-CoV-2 infection. Increasing age, low baseline lymphocyte count and the presence of co-morbidities are additional risk factors for poor outcome in this cohort.

\section{Introduction}

The risk of morbidity and mortality following SARS-CoV-2 infection in patients with primary immunodeficiency (PID) and secondary immunodeficiency (SID) remains unclear. National epidemiological studies tend to consider immunocompromised individuals as a homogenous group (e.g., HIV-1 infection, solid organ transplant, immunosuppressive medications) and are unable to inform our understanding of outcome in individuals with rare diseases [1, 2].

Instead, retrospective case series have been used to develop our understanding of the risk in patients with PID and SID [3-9]. While the results of such studies must be interpreted with caution, a consistent pattern has emerged showing that adult patients with PID and SID are at an increased risk of morbidity and mortality from COVID-19 compared to the general population, and that an increased prevalence of chronic co-morbidities, potentially driven by underlying immunodeficiency, partially contributes to that risk. The risk of severe morbidity and mortality from COVID-19 in children with PID or SID remains less clear with data from the UK showing no increased risk in this group [7], although mortality has been reported elsewhere [8].

In order to more comprehensively understand the risk of morbidity and mortality from COVID-19 in patients with PID and SID, the United Kingdom Primary Immunodeficiency Network (UK PIN) has systematically collated outcomes from COVID-19 in patients with PID and SID throughout the pandemic. This national effort has enabled a large cohort of individuals with PID and SID to be developed, and their outcomes understood in comparison to well curated national statistics.

Herein, we report morbidity and mortality from COVID-19 in a cohort of 310 individuals with PID or SID from the United Kingdom; we build upon our previous work [5] by providing revised estimates of mortality in clinically important PID and SID subgroups, insight into independent risk factors for mortality and the efficacy of targeted COVID-19 treatments in this cohort.

\section{Methods}

The United Kingdom Primary Immunodeficiency Network (UK PIN) has systematically collected data from its members on the outcomes of SARS-CoV-2 infection in patients under the care of Clinical Immunology teams across the United Kingdom since March 2020.

In March 2020, data collection proformas were sent to all UK PIN affiliated pediatric and adult immunologists and centres by email. During the first wave of the UK pandemic (March 2020 - July 2020), data collected included age, sex, ethnicity, body mass index, pre-infection lymphocyte count (taken from a full blood count at the last immunology outpatient appointment prior to SARS-CoV-2 infection), prior immunological treatments [e.g. immunoglobulin replacement, immunosuppression (any biologic immunosuppressive within 1 month of SARS-CoV-2 infection, any anti-CD20 depleting agents within 6-months of SARSCoV-2 infection, or any daily oral immunosuppression including steroids), and antibiotic prophylaxis], existing chronic comorbidities, SARS-CoV-2 PCR result, whether individuals were hospitalized, and whether individuals survived or died. The outcomes from the first 100 cases in this case series have been published previously [5]. During the subsequent waves of the UK pandemic (September 2020 - present), additional data collected included whether an individual received 
any targeted COVID-19 treatments during their inpatient stay and whether an individual seroconverted following natural infection. The published literature was also reviewed to capture any further UK cases of COVID-19 in immunodeficiency patients that had been published elsewhere: this literature search revealed two additional cases $[10,11]$ which have been included.

Data were collated according to 2019 IUIS classification of inborn errors of immunity and further analysis undertaken on three subgroups: i) individuals with common variable immunodeficiency (including six patients with monogenic CVID-like disease secondary to haploinsufficiency of NFKB1, NFKB2, BACH-2 or

CTLA-4), collectively referred to as CVID-phenotype, ii) all individuals with primary immunodeficiency receiving immunoglobulin replacement (IgRT) including CVID but excluding those who had received definitive treatment with allogenic stem cell transplantation or gene therapy (i.e. those with a clinically significant antibody deficiency regardless of proximal diagnosis) and iii) individuals with secondary immunodeficiency.

Data were analyzed using GraphPad Prism 9.0 (GraphPad Prism Software, San Diego, Calif). Differences between the distributions of continuous variables were evaluated using the 2-tailed Mann-Whitney U test. Differences between categorical variables were evaluated using the 2-tailed Chi-square test. Odds ratios were calculated using the Baptista-Pike method. Case fatality ratio (CFR) is defined as the ratio between total deaths and total PCR-proven infections. Infection fatality ratio (IFR) is defined as the ratio between total deaths and total suspected or proven SARS-CoV-2 infections. UK national statistics are sourced from the UK Department of Health and Social Care (1/1/2020-29/3/2021). When analyzing pre-SARS-CoV-2 infection lymphocyte counts in the SID cohort, patients with chronic lymphocytic leukemia were excluded.

Multiple logistic regression models were constructed using survival as the outcome variable. Age and baseline lymphocyte count were included as continuous variables; sex, receipt of prophylactic antibiotics, receipt of immune suppression, and the presence of different comorbidities were included as categorical variables. Odds ratios for continuous variables are expressed as the change in odds of survival per unit increase of that continuous variable within the model. Odds ratios for categorical variables are expressed as the odds ratio associated with mortality if that comorbidity is present.

\section{Results}

A total of 310 cases of SARS-CoV-2 infection in patients with PID or SID were recorded between March 2020 and July 2021 from 27 UK PIN affiliated immunology departments representing all four nations of the United Kingdom. Cases included 218 individuals with primary immunodeficiency, 6 of whom had received allogenic stem cell transplantation or gene therapy for their underlying condition, 92 individuals with secondary immunodeficiency. In addition, 13 individuals with $\mathrm{C} 1$ inhibitor deficiency and 3 with autoinflammatory diseases were also reported.

In patients with primary or secondary immunodeficiency, $45.8 \%(n=142 / 310)$ of patients were hospitalised and the overall IFR in this cohort was $17.7 \%$ ( $n=$ 55/310). 86.5\% ( $n=268 / 310)$ had SARS-CoV-2 confirmed by PCR or rapid antigen testing; the remainder had either an illness consistent with COVID-19 when the availability of molecular testing for SARS-CoV-2 was limited and had the diagnosis made clinically or radiologically. Hospitalisation rates, IFR and CFR data for specific immunodeficiencies are presented in Table 1. 
Table 1

Overall description of UK PIN COVID-19 cohort.

\begin{tabular}{|c|c|c|c|c|c|c|c|c|c|c|c|c|}
\hline Diagnosis & $\mathbf{n}$ & $\begin{array}{l}\text { Age } \\
\text { (y) }\end{array}$ & $\begin{array}{l}\text { Sexn } \\
(\% F)\end{array}$ & $\begin{array}{l}\text { Ethnicity } \\
\text { n } \\
\text { (\%BAME) }\end{array}$ & $\begin{array}{l}\text { PCR/antigen } \\
\text { proven } \\
\text { infection }\end{array}$ & IgRT & pAbx & $\begin{array}{l}\text { Immune } \\
\text { suppression }\end{array}$ & Hospitalised & Deaths & \%IFR & $\%$ CFF \\
\hline \multicolumn{13}{|c|}{ Predominant antibody deficiencies } \\
\hline $\begin{array}{l}\text { Common variable } \\
\text { immunodeficiency } \\
\text { (genetically } \\
\text { undifferentiated) }\end{array}$ & 87 & $\begin{array}{l}49 \\
(30.7- \\
58.3)\end{array}$ & $\begin{array}{l}51 \\
(58.6)\end{array}$ & $\begin{array}{l}4 \\
(5.3)\end{array}$ & $\begin{array}{l}80 \\
(92.0)\end{array}$ & $\begin{array}{l}76 \\
(87.3)\end{array}$ & $\begin{array}{l}45 \\
(51.7)\end{array}$ & $\begin{array}{l}16 \\
(18.3)\end{array}$ & $\begin{array}{l}38 \\
(43.7)\end{array}$ & 15 & 17.2 & 18.8 \\
\hline $\begin{array}{l}\text { NFKB1 } \\
\text { haploinsufficiency* }\end{array}$ & 2 & $\begin{array}{l}34 \\
(27.0- \\
41.0)\end{array}$ & $\begin{array}{l}0 \\
(0.0)\end{array}$ & $\begin{array}{l}0 \\
(0.0)\end{array}$ & $\begin{array}{l}1 \\
(50.0)\end{array}$ & $\begin{array}{l}2 \\
(100.0)\end{array}$ & $\begin{array}{l}2 \\
(100.0)\end{array}$ & $\begin{array}{l}1 \\
(50.0)\end{array}$ & $\begin{array}{l}1 \\
(50.0)\end{array}$ & 0 & 0.0 & 0.0 \\
\hline $\begin{array}{l}\text { BACH2 } \\
\text { haploinsufficiency* }\end{array}$ & 2 & $\begin{array}{l}38 \\
(22.0- \\
55.0)\end{array}$ & $\begin{array}{l}1 \\
(50.0)\end{array}$ & $\begin{array}{l}0 \\
(0.0)\end{array}$ & $\begin{array}{l}2 \\
(100.0)\end{array}$ & $\begin{array}{l}2 \\
(50.0)\end{array}$ & $\begin{array}{l}0 \\
(0.0)\end{array}$ & $\begin{array}{l}0 \\
(0.0)\end{array}$ & $\begin{array}{l}0 \\
(0.0)\end{array}$ & 0 & 0.0 & 0.0 \\
\hline $\begin{array}{l}\text { NFKB2 } \\
\text { haploinsufficiency* }\end{array}$ & 1 & Adult & $\begin{array}{l}1 \\
(100.0)\end{array}$ & $\begin{array}{l}0 \\
(0.0)\end{array}$ & $\begin{array}{l}1 \\
(100.0)\end{array}$ & $\begin{array}{l}1 \\
(100.0)\end{array}$ & $\begin{array}{l}1 \\
(100.0)\end{array}$ & $\begin{array}{l}0 \\
(0.0)\end{array}$ & $\begin{array}{l}1 \\
(100.0)\end{array}$ & 1 & 100.0 & 100.0 \\
\hline $\begin{array}{l}\text { CTLA-4 } \\
\text { haploinsufficiency* }\end{array}$ & 1 & Adult & $0(0.0)$ & $\begin{array}{l}1 \\
(100.0)\end{array}$ & $\begin{array}{l}1 \\
(100.0)\end{array}$ & $\begin{array}{l}1 \\
(100.0)\end{array}$ & $\begin{array}{l}1 \\
(100.0)\end{array}$ & $\begin{array}{l}1 \\
(100.0)\end{array}$ & $\begin{array}{l}1 \\
(100.0)\end{array}$ & 1 & 100.0 & 100.0 \\
\hline XLA & 26 & $\begin{array}{l}29.5 \\
(21.3- \\
33.8)\end{array}$ & $\begin{array}{l}0 \\
(100.0)\end{array}$ & $\begin{array}{l}6 \\
(23.1)\end{array}$ & $\begin{array}{l}22 \\
(84.6)\end{array}$ & $\begin{array}{l}26 \\
(100.0)\end{array}$ & $\begin{array}{l}16 \\
(61.5)\end{array}$ & $\begin{array}{l}0 \\
(0.0)\end{array}$ & $\begin{array}{l}11 \\
(42.3)\end{array}$ & 2 & 7.7 & 9.1 \\
\hline $\begin{array}{l}\text { Undifferentiated } \\
\text { primary antibody } \\
\text { deficiency }\end{array}$ & 26 & $\begin{array}{l}52.0 \\
(30.3- \\
72.5)\end{array}$ & $\begin{array}{l}18 \\
(69.2)\end{array}$ & $\begin{array}{l}1 \\
\text { (3.8) }\end{array}$ & $\begin{array}{l}20 \\
(76.9)\end{array}$ & $\begin{array}{l}14 \\
(53.8)\end{array}$ & $\begin{array}{l}12 \\
(46.2)\end{array}$ & $\begin{array}{l}2 \\
\\
(7.7)\end{array}$ & $\begin{array}{l}6 \\
(23.1)\end{array}$ & 4 & 15.4 & 20.0 \\
\hline SPAD & 8 & $\begin{array}{l}57 \\
(51.5- \\
69.8)\end{array}$ & $\begin{array}{l}3 \\
(37.5)\end{array}$ & $\begin{array}{l}1 \\
(14.3)\end{array}$ & $\begin{array}{l}6 \\
(75.0)\end{array}$ & $\begin{array}{l}6 \\
(75.0)\end{array}$ & $\begin{array}{l}6 \\
(75.0)\end{array}$ & $\begin{array}{l}1 \\
(12.5)\end{array}$ & $\begin{array}{l}5 \\
(62.5)\end{array}$ & 2 & 25.0 & 33.3 \\
\hline Good's Syndrome & 5 & $\begin{array}{l}72 \\
(60.5- \\
77.5)\end{array}$ & $\begin{array}{l}3 \\
(60.0)\end{array}$ & $\begin{array}{l}0 \\
(0.0)\end{array}$ & $\begin{array}{l}5 \\
(100.0)\end{array}$ & $\begin{array}{l}5 \\
(100.0)\end{array}$ & $\begin{array}{l}5 \\
(100.0)\end{array}$ & $\begin{array}{l}0 \\
(0.0)\end{array}$ & $\begin{array}{l}4 \\
(80.0)\end{array}$ & 1 & 20.0 & 20.0 \\
\hline Evans Syndrome & 3 & $\begin{array}{l}22.0 \\
(19.0- \\
55.0)\end{array}$ & $\begin{array}{l}2 \\
(66.7)\end{array}$ & $\begin{array}{l}0 \\
(0.0)\end{array}$ & $\begin{array}{l}2 \\
(66.7)\end{array}$ & $\begin{array}{l}2 \\
(66.7)\end{array}$ & $\begin{array}{l}1 \\
(33.3)\end{array}$ & $\begin{array}{l}2 \\
(66.7)\end{array}$ & $\begin{array}{l}1 \\
(33.3)\end{array}$ & 0 & 0.0 & 0.0 \\
\hline \multicolumn{13}{|c|}{ Combined immunodeficiencies } \\
\hline $\begin{array}{l}\text { Hyper lgE } \\
\text { syndrome (STAT3) }\end{array}$ & 2 & $\begin{array}{l}36 \\
(26.0- \\
46.0)\end{array}$ & $\begin{array}{l}0 \\
(0.0)\end{array}$ & $\begin{array}{l}0 \\
(0.0)\end{array}$ & $\begin{array}{l}2 \\
(100.0)\end{array}$ & $\begin{array}{l}1 \\
(50.0)\end{array}$ & $\begin{array}{l}1 \\
(50.0)\end{array}$ & $\begin{array}{l}0 \\
(0.0)\end{array}$ & $\begin{array}{l}1 \\
(50.0)\end{array}$ & 0 & 0.0 & 0.0 \\
\hline $\begin{array}{l}\text { Hyper lgM } \\
\text { syndrome ( } 2 \mathrm{XL} \text {, } \\
\text { 1AID) }\end{array}$ & 3 & $\begin{array}{l}23 \\
(17.0- \\
40.0)\end{array}$ & $\begin{array}{l}0 \\
(0.0)\end{array}$ & $\begin{array}{l}2 \\
(66.7)\end{array}$ & $\begin{array}{l}3 \\
(100.0)\end{array}$ & $\begin{array}{l}3 \\
(100.0)\end{array}$ & $\begin{array}{l}2 \\
(66.6)\end{array}$ & $\begin{array}{l}0 \\
(0.0)\end{array}$ & $\begin{array}{l}0 \\
(0.0)\end{array}$ & 0 & 0.0 & 0.0 \\
\hline $\begin{array}{l}\text { Wiskott Aldrich } \\
\text { syndrome }\end{array}$ & 3 & $\begin{array}{l}37.0 \\
(34.0- \\
39.0)\end{array}$ & $\begin{array}{l}0 \\
(0.0)\end{array}$ & $\begin{array}{l}1 \\
(33.3)\end{array}$ & $\begin{array}{l}3 \\
(100.0)\end{array}$ & $\begin{array}{l}3 \\
(100.0)\end{array}$ & $\begin{array}{l}2 \\
(66.6)\end{array}$ & $\begin{array}{l}0 \\
(0.0)\end{array}$ & $\begin{array}{l}0 \\
(0.0)\end{array}$ & 0 & 0.0 & 0.0 \\
\hline $\begin{array}{l}\text { Undifferentiated } \\
\text { CID }\end{array}$ & 8 & $\begin{array}{l}43.0 \\
(29.0- \\
53.8)\end{array}$ & $\begin{array}{l}6 \\
(75.0)\end{array}$ & $\begin{array}{l}2 \\
(25.0)\end{array}$ & $\begin{array}{l}5 \\
(62.5)\end{array}$ & $\begin{array}{l}6 \\
(75.0)\end{array}$ & $\begin{array}{l}7 \\
(87.5)\end{array}$ & $\begin{array}{l}2 \\
(25.0)\end{array}$ & $\begin{array}{l}3 \\
(37.5)\end{array}$ & 2 & 25.0 & 40.0 \\
\hline ICOS deficiency & 2 & $\begin{array}{l}32.5 \\
(28.0- \\
37.0)\end{array}$ & $\begin{array}{l}2 \\
(100.0)\end{array}$ & $\begin{array}{l}1 \\
(50.0)\end{array}$ & $\begin{array}{l}2 \\
(100.0)\end{array}$ & $\begin{array}{l}1 \\
(50.0)\end{array}$ & $\begin{array}{l}1 \\
(50.0)\end{array}$ & $\begin{array}{l}1 \\
(50.0)\end{array}$ & $\begin{array}{l}1 \\
(50.0)\end{array}$ & 0 & 0.0 & 0.0 \\
\hline
\end{tabular}

Individuals with monogenic CVID-like disease are listed separately and marked with an asterisks. The chronic granulomatous disease (CGD) group included 2 individuals with autosomal recessive CGD, 1 individual with X-linked CGD and 1 symptomatic carrier. Bone marrow transplantation/gene therapy group

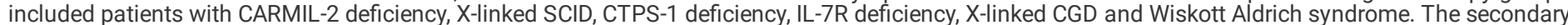

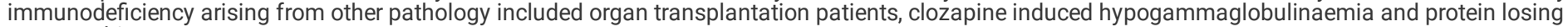
enteropathies. 


\begin{tabular}{|c|c|c|c|c|c|c|c|c|c|c|c|c|}
\hline Diagnosis & $\mathrm{n}$ & $\begin{array}{l}\text { Age } \\
\text { (y) }\end{array}$ & $\begin{array}{l}\text { Sex } n \\
(\% F)\end{array}$ & $\begin{array}{l}\text { Ethnicity } \\
\text { n } \\
\text { (\%BAME) }\end{array}$ & $\begin{array}{l}\text { PCR/antigen } \\
\text { proven } \\
\text { infection }\end{array}$ & IgRT & pAbx & $\begin{array}{l}\text { Immune } \\
\text { suppression }\end{array}$ & Hospitalised & Deaths & \%IFR & \%CFF \\
\hline \multirow{2}{*}{$\begin{array}{l}\text { Idiopathic T cell } \\
\text { lymphopaenia }\end{array}$} & \multirow[t]{2}{*}{1} & \multirow[t]{2}{*}{ Adult } & \multirow{2}{*}{$\begin{array}{l}1 \\
(100.0)\end{array}$} & 0 & 0 & \multirow[t]{2}{*}{$0(0.0)$} & \multirow{2}{*}{$\begin{array}{l}1 \\
(100.0)\end{array}$} & 0 & 0 & \multirow[t]{2}{*}{0} & \multirow[t]{2}{*}{0.0} & \multirow[t]{2}{*}{0.0} \\
\hline & & & & $(0.0)$ & $(0.0)$ & & & $(0.0)$ & $(0.0)$ & & & \\
\hline \multirow[t]{2}{*}{ GATA-2 } & \multirow[t]{2}{*}{1} & \multirow[t]{2}{*}{ Adult } & \multirow{2}{*}{$\begin{array}{l}1 \\
(100.0)\end{array}$} & 0 & 1 & \multirow[t]{2}{*}{$0(0.0)$} & \multirow[t]{2}{*}{$0(0.0)$} & 0 & 1 & \multirow[t]{2}{*}{0} & \multirow[t]{2}{*}{0.0} & \multirow[t]{2}{*}{0.0} \\
\hline & & & & $(0.0)$ & (100.0) & & & $(0.0)$ & $(100.0)$ & & & \\
\hline \multirow[t]{2}{*}{ TPP2 deficiency } & \multirow[t]{2}{*}{1} & \multirow[t]{2}{*}{ Child } & \multirow{2}{*}{$\begin{array}{l}1 \\
(100.0)\end{array}$} & 1 & 1 & \multirow[t]{2}{*}{$0(0.0)$} & 1 & 1 & 1 & 0 & 0.0 & 0.0 \\
\hline & & & & $(100.0)$ & (100.0) & & & $(100.0)$ & $(100.0)$ & & & \\
\hline $\begin{array}{l}\text { CARD11 dominant } \\
\text { negative }\end{array}$ & 2 & $\begin{array}{l}21.5 \\
(18.0-\end{array}$ & 1 & 0 & 2 & $0(0.0)$ & 1 & 0 & 1 & 0 & 0.0 & 0.0 \\
\hline & & & & $(0.0)$ & $(100.0)$ & & & $(0.0)$ & $(100.0)$ & & & \\
\hline Syndromic CID & & & & & & & & & & & & \\
\hline Ataxia & 4 & 11 & 3 & 3 & 4 & 0 & 2 & 1 & 0 & 0 & 0.0 & 0.0 \\
\hline & & $12.8)$ & & $(100.0)$ & $(100.0)$ & $(0.0)$ & & $(25.0)$ & $(0.0)$ & & & \\
\hline Trisomy 21 & 1 & Adult & 1 & 0 & 1 & 1 & 1 & $0(0.0)$ & 1 & 1 & 100.0 & 100.0 \\
\hline & & & & $(0.0)$ & $(100.0)$ & & & & $(100.0)$ & & & \\
\hline 22q11 & 2 & 19.5 & 0 & 0 & 2 & 0 & 0 & 0 & 1 & 0 & 0.0 & 0.0 \\
\hline & & $21.0)$ & $(0.0)$ & $(0.0)$ & $(100.0)$ & $(0.0)$ & $(0.0)$ & $(0.0)$ & $(50.0)$ & & & \\
\hline Kabuki's syndrome & 1 & Adult & 0 & 0 & 0 & 1 & 1 & 0 & 1 & 0 & 0.0 & 0.0 \\
\hline & & & $(0.0)$ & $(0.0)$ & $(0.0)$ & & & $(0.0)$ & $(100.0)$ & & & \\
\hline $\begin{array}{l}\text { Syndromic } \\
\text {. }\end{array}$ & 1 & Adult & 0 & 0 & 1 & 1 & 0 & 0 & 0 & 0 & 0.0 & 0.0 \\
\hline $\begin{array}{l}\text { lmmunodeficiency } \\
\text { with defect in DNA } \\
\text { damage repair }\end{array}$ & & & $(0.0)$ & $(0.0)$ & $(100.0)$ & & $(0.0)$ & $(0.0)$ & $(0.0)$ & & & \\
\hline Neutrophil defects & & & & & & & & & & & & \\
\hline $\begin{array}{l}\text { Chronic } \\
\text {. }\end{array}$ & 4 & 26.5 & 3 & 2 & 4 & 1 & 3 & 0 & 1 & 0 & 0.0 & 0.0 \\
\hline $\begin{array}{l}\text { granulomatous } \\
\text { disease }\end{array}$ & & $\begin{array}{l}(8.0- \\
42.8)\end{array}$ & & $(50.0)$ & (100.0) & & & $(0.0)$ & $(25.0)$ & & & \\
\hline Immunodeficiencies & follor & ing bone & narrow tra & splantation & or gene therapy & & & & & & & \\
\hline Post bone marrow & 6 & 27 & 3 & 1 & 5 & 3 & 3 & 1 & 1 & 0 & 0.0 & 0.0 \\
\hline $\begin{array}{l}\text { therapy } \\
\text { the }\end{array}$ & & $31.3)$ & & (16.7) & (83.3) & & & (16.7) & $(16.7)$ & & & \\
\hline Disorders of immun & regu & tion & & & & & & & & & & \\
\hline XLP & 2 & 30 & 0 & 0 & 2 & 1 & 2 & 1 & 1 & 0 & 0.0 & 0.0 \\
\hline & & $37.0)$ & $(0.0)$ & $(0.0)$ & $(100.0)$ & & & $(50.0)$ & $(50.0)$ & & & \\
\hline ALPS & 3 & 19 & 3 & 0 & 2 & 1 & 1 & 2 & 0 & 0 & 0.0 & 0.0 \\
\hline & & $23.0)$ & & $(0.0)$ & (66.6) & & & $(66.7)$ & $(0.0)$ & & & \\
\hline STAT3 GoF & 1 & Adult & 0 & 1 & 0 & $0(0.0)$ & 1 & 0 & 0 & 0 & 0.0 & 0.0 \\
\hline & & & $(0.0)$ & $(100.0)$ & $(0.0)$ & & & $(0.0)$ & $(0.0)$ & & & \\
\hline Complement deficie & cies & & & & & & & & & & & \\
\hline C1 inhibitor & 13 & 45 & 6 & 1 & 9 & $\mathrm{n} / \mathrm{a}$ & $\mathrm{n} / \mathrm{a}$ & $\mathrm{n} / \mathrm{a}$ & 1 & 0 & 0.0 & 0.0 \\
\hline & & $51.5)$ & & (7.7) & $(69.2)$ & & & & (7.7) & & & \\
\hline MBL deficiency & 2 & 56.0 & 2 & 0 & 2 & 1 & 0 & 0 & 2 & 0 & 0.0 & 0.0 \\
\hline & & $66.0)$ & & $(0.0)$ & (100.0) & & $(0.0)$ & $(0.0)$ & $(100.0)$ & & & \\
\hline TCC deficiency & 2 & 39.5 & 1 & 0 & 2 & 0 & 2 & 0 & 1 & 1 & 50.0 & 50.0 \\
\hline & & $59.0)$ & & $(0.0)$ & (100.0) & $(0.0)$ & & $(0.0)$ & $(50.0)$ & & & \\
\hline
\end{tabular}

Individuals with monogenic CVID-like disease are listed separately and marked with an asterisks. The chronic granulomatous disease (CGD) group included 2 individuals with autosomal recessive CGD, 1 individual with X-linked CGD and 1 symptomatic carrier. Bone marrow transplantation/gene therapy group

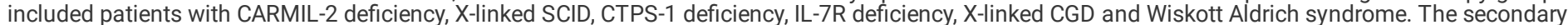

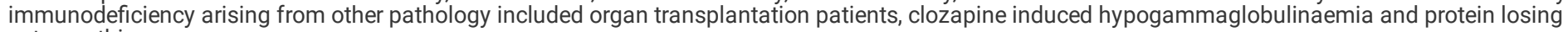
enteropathies. 


\begin{tabular}{|c|c|c|c|c|c|c|c|c|c|c|c|c|}
\hline Diagnosis & $\mathbf{n}$ & $\begin{array}{l}\text { Age } \\
\text { (v) }\end{array}$ & $\begin{array}{l}\text { Sexn } \\
(\% F)\end{array}$ & $\begin{array}{l}\text { Ethnicity } \\
\text { n } \\
\text { (\%BAME) }\end{array}$ & $\begin{array}{l}\text { PCR/antigen } \\
\text { proven } \\
\text { infection }\end{array}$ & IgRT & pAbx & $\begin{array}{l}\text { Immune } \\
\text { suppression }\end{array}$ & Hospitalised & Deaths & \%IFR & \%CFF \\
\hline \multicolumn{13}{|c|}{ Other primary immunodeficiency } \\
\hline $\begin{array}{l}\text { Undifferentiated } \\
\text { PID }\end{array}$ & 5 & $\begin{array}{l}43.0 \\
(18.0- \\
58.0)\end{array}$ & $\begin{array}{l}2 \\
(40.0)\end{array}$ & $\begin{array}{l}0 \\
(0.0)\end{array}$ & $\begin{array}{l}4 \\
(80.0)\end{array}$ & $\begin{array}{l}1 \\
(20.0)\end{array}$ & $\begin{array}{l}1 \\
(20.0)\end{array}$ & $\begin{array}{l}0 \\
(0.0)\end{array}$ & $\begin{array}{l}1 \\
(25.0)\end{array}$ & 0 & 0.0 & 0.0 \\
\hline \multicolumn{13}{|c|}{ Autoinflammatory disease/interferonopathies } \\
\hline $\begin{array}{l}\text { Hyper lgD } \\
\text { syndrome }\end{array}$ & 1 & Adult & $\begin{array}{l}1 \\
(100.0)\end{array}$ & $\begin{array}{l}1 \\
(100.0)\end{array}$ & $\begin{array}{l}0 \\
(0.0)\end{array}$ & $\begin{array}{l}0 \\
(0.0)\end{array}$ & $\begin{array}{l}0 \\
(0.0)\end{array}$ & $\begin{array}{l}1 \\
(100.0)\end{array}$ & $\begin{array}{l}0 \\
(0.0)\end{array}$ & 0 & 0.0 & 0.0 \\
\hline $\begin{array}{l}\text { Aicardi-Gouteres } \\
\text { syndrome }\end{array}$ & 1 & Child & $\begin{array}{l}1 \\
(100.0)\end{array}$ & $\begin{array}{l}1 \\
(100.0)\end{array}$ & $\begin{array}{l}1 \\
(100.0)\end{array}$ & $\begin{array}{l}0 \\
(0.0)\end{array}$ & $\begin{array}{l}0 \\
(0.0)\end{array}$ & $\begin{array}{l}0 \\
(0.0)\end{array}$ & $\begin{array}{l}0 \\
(0.0)\end{array}$ & 0 & 0.0 & 0.0 \\
\hline $\begin{array}{l}\text { A20 } \\
\text { haploinsufficiency }\end{array}$ & 1 & Child & $\begin{array}{l}1 \\
(100.0)\end{array}$ & $\begin{array}{l}0 \\
(0.0)\end{array}$ & $\begin{array}{l}1 \\
(100.0)\end{array}$ & $\begin{array}{l}1 \\
(100.0)\end{array}$ & $\begin{array}{l}1 \\
(100.0)\end{array}$ & $\begin{array}{l}1 \\
(100.0)\end{array}$ & $\begin{array}{l}1 \\
(100.0)\end{array}$ & 0 & 0.0 & 0.0 \\
\hline Secondary immunoc & eficiel & & & & & & & & & & & \\
\hline $\begin{array}{l}\text { Secondary to } \\
\text { haematological } \\
\text { disease }\end{array}$ & 52 & $\begin{array}{l}65.0 \\
(56.3- \\
74.8)\end{array}$ & $\begin{array}{l}25 \\
(48.1)\end{array}$ & $\begin{array}{l}6 \\
(11.5)\end{array}$ & $\begin{array}{l}46 \\
(88.4)\end{array}$ & $\begin{array}{l}37 \\
(71.2)\end{array}$ & $\begin{array}{l}27 \\
(51.9)\end{array}$ & $\begin{array}{l}15 \\
(31.9)\end{array}$ & $\begin{array}{l}29 \\
(55.8)\end{array}$ & 12 & 23.1 & 26.1 \\
\hline $\begin{array}{l}\text { Secondary to } \\
\text { rheumatological } \\
\text { disease }\end{array}$ & 21 & $\begin{array}{l}58.0 \\
(50.0- \\
74.0)\end{array}$ & $\begin{array}{l}18 \\
(85.7)\end{array}$ & $\begin{array}{l}4 \\
(19.0)\end{array}$ & $\begin{array}{l}21 \\
(100.0)\end{array}$ & $\begin{array}{l}15 \\
(71.4)\end{array}$ & $\begin{array}{l}12 \\
(57.1)\end{array}$ & $\begin{array}{l}14 \\
(77.8)\end{array}$ & $\begin{array}{l}17 \\
(81.0)\end{array}$ & 8 & 38.1 & 38.1 \\
\hline $\begin{array}{l}\text { Secondary to } \\
\text { respiratory disease }\end{array}$ & 6 & $\begin{array}{l}67.0 \\
(50.0- \\
79.3)\end{array}$ & $\begin{array}{l}4 \\
(66.7)\end{array}$ & $\begin{array}{l}0 \\
(0.0)\end{array}$ & $\begin{array}{l}5 \\
(83.3)\end{array}$ & $\begin{array}{l}3 \\
(50.0)\end{array}$ & $\begin{array}{l}6 \\
(100.0)\end{array}$ & $\begin{array}{l}4 \\
(66.7)\end{array}$ & $\begin{array}{l}4 \\
(66.7)\end{array}$ & 3 & 50.0 & 60.0 \\
\hline $\begin{array}{l}\text { Secondary to other } \\
\text { pathology or not } \\
\text { specified }\end{array}$ & 13 & $\begin{array}{l}57.0 \\
(27.0- \\
67.5)\end{array}$ & $\begin{array}{l}6 \\
(46.2)\end{array}$ & $\begin{array}{l}3 \\
(23.1)\end{array}$ & $\begin{array}{l}11 \\
(84.6)\end{array}$ & $\begin{array}{l}7 \\
(53.8)\end{array}$ & $\begin{array}{l}10 \\
(76.9)\end{array}$ & $\begin{array}{l}4 \\
(36.3)\end{array}$ & $\begin{array}{l}6 \\
(46.2)\end{array}$ & 2 & 15.4 & 18.2 \\
\hline $\begin{array}{l}\text { Individuals with mor } \\
2 \text { individuals with au } \\
\text { included patients wi } \\
\text { immunodeficiency a } \\
\text { enteropathies. }\end{array}$ & geni & CVID-like & $\begin{array}{l}\text { lisease a } \\
\text { CGD, } 1 \\
\text { ency, X-I } \\
\text { atholog. }\end{array}$ & $\begin{array}{l}\text { listed sepa } \\
\text { ividual wit } \\
\text { ed SCID, C } \\
\text { icluded org }\end{array}$ & $\begin{array}{l}\text { Itely and mark } \\
\text { X-linked CGD a } \\
\text { SS-1 deficiency } \\
\text { n transplantati }\end{array}$ & $\begin{array}{l}\text { d with an } \\
\text { id } 1 \text { sym } \\
\text { IL-7R def } \\
\text { in patient }\end{array}$ & $\begin{array}{l}\text { sterisks. } \\
\text { omatic ca } \\
\text { ency, X-li } \\
\text { clozapin }\end{array}$ & $\begin{array}{l}\text { he chronic grar } \\
\text { rier. Bone marr } \\
\text { ked CGD and } V \\
\text { induced hypog }\end{array}$ & $\begin{array}{l}\text { ulomatous dis } \\
\text { w transplanta } \\
\text { iskott Aldrich } \\
\text { ammaglobulin }\end{array}$ & $\begin{array}{l}\text { se (CGD) } \\
\text { n/gene tl } \\
\text { idrome. } \\
\text { mia and }\end{array}$ & $\begin{array}{l}\text { group ir } \\
\text { erapy } \mathrm{g} \\
\text { he seco } \\
\text { rotein I }\end{array}$ & $\begin{array}{l}\text { luded } \\
\text { up } \\
\text { dary } \\
\text { ing }\end{array}$ \\
\hline
\end{tabular}

To understand risk factors associated with mortality from COVID-19 in patients with immunodeficiency, the following sub-groups of individuals were considered: individuals with common variable immunodeficiency including those with monogenic CVID-like disease, collectively referred to as CVID-phenotype $(n=93)$, individuals with PID receiving immunoglobulin replacement therapy $(n=159)$, and individuals with secondary immunodeficiency $(n=92)$.

\section{Common variable immunodeficiency disorder}

Ninety-three cases of SARS-CoV-2 infection in patients with a CVID-phenotype were analysed; 87 individuals had genetically undifferentiated CVID and 6 had a monogenic CVID-like disease (Table 1). The median age of this cohort was 48 years (IQR 30.3-57.0), 57.0\% $(n=53 / 93)$ were female and $51.6 \%(n=48 / 93)$ were receiving antibiotic prophylaxis. IFR in this cohort was $18.3 \%(n=17 / 93)$ and increased CFR were observed in all age brackets over 20 years in comparison to national statistics (Table 2). Median age of death from COVID-19 in individuals with CVID was 63.0 years. 
Age stratified mortality within major subgroups of the UK PIN COVID-19 cohort

\begin{tabular}{|c|c|c|c|c|c|c|c|c|c|c|c|c|c|c|c|c|c|}
\hline & PID & ith antibc & dy deficiency ( & = 159) & & & CVI & phenc & ype $(n=9$ & & & & Syn & ptomatic & SID $(n=92$ & & \\
\hline Age & $\mathrm{N}$ & PCR+ & $\begin{array}{l}\text { Hospitalized } \\
(\mathrm{n}, \%)\end{array}$ & Deaths & $\begin{array}{l}\text { IFR } \\
(\%)\end{array}$ & $\begin{array}{l}\text { CFR } \\
(\%)\end{array}$ & $\mathrm{N}$ & $\begin{array}{l}\text { PCR } \\
+\end{array}$ & $\begin{array}{l}\text { Hospit } \\
\text { alized } \\
(n, \%)\end{array}$ & Deaths & $\begin{array}{l}\text { IFR } \\
(\%)\end{array}$ & $\begin{array}{l}\text { CFR } \\
(\%)\end{array}$ & $\mathrm{N}$ & PCR+ & $\begin{array}{l}\text { Hospital- } \\
\text { ized } \\
(n, \%)\end{array}$ & Deaths & $\begin{array}{l}\text { If } \\
(c\end{array}$ \\
\hline $0-9$ & 4 & 4 & $\begin{array}{l}1 \\
(25.0)\end{array}$ & 0 & 0.0 & 0.0 & - & - & - & - & - & - & - & - & - & - & - \\
\hline $\begin{array}{l}10- \\
19\end{array}$ & 8 & 7 & $\begin{array}{l}2 \\
(25.0)\end{array}$ & 0 & 0.0 & 0.0 & 1 & 1 & $\begin{array}{l}0 \\
(0.0)\end{array}$ & 0 & 0 & 0.0 & 1 & 1 & $\begin{array}{l}0 \\
(0.0)\end{array}$ & 0 & 0 \\
\hline $\begin{array}{l}20- \\
29\end{array}$ & 33 & 28 & $\begin{array}{l}6 \\
(18.2)\end{array}$ & 1 & 3.0 & 3.6 & 20 & 18 & $\begin{array}{l}4 \\
(20.0)\end{array}$ & 1 & 5.0 & 5.6 & 5 & 1 & $\begin{array}{l}2 \\
(40.0)\end{array}$ & 0 & 0 \\
\hline $\begin{array}{l}30- \\
39\end{array}$ & 33 & 25 & $10^{*}(30.3)$ & 1 & 3.0 & 4.0 & 16 & 14 & $\begin{array}{l}6^{*} \\
(37.5)\end{array}$ & 1 & 6.3 & 7.1 & 5 & 5 & $\begin{array}{l}0 \\
(0.0)\end{array}$ & 0 & 0 \\
\hline $\begin{array}{l}40- \\
49\end{array}$ & 21 & 20 & $\begin{array}{l}12 \\
(57.1)\end{array}$ & 3 & 14.2 & 15.0 & 14 & 13 & $\begin{array}{l}7 \\
(50.0)\end{array}$ & 1 & 7.1 & 7.7 & 6 & 6 & $\begin{array}{l}4 \\
(66.6)\end{array}$ & 4 & 6 \\
\hline $\begin{array}{l}50- \\
59\end{array}$ & 25 & 23 & $\begin{array}{l}12 \\
(48.0)\end{array}$ & 9 & 36.0 & 39.1 & 21 & 18 & $\begin{array}{l}8 \\
(38.1)\end{array}$ & 5 & 23.8 & 27.8 & 20 & 18 & $\begin{array}{l}16 \\
(80.0)\end{array}$ & 5 & 2 \\
\hline $\begin{array}{l}60- \\
69\end{array}$ & 14 & 13 & $\begin{array}{l}5^{\$} \\
(35.7)\end{array}$ & 3 & 21.4 & 23.1 & 7 & 7 & $\begin{array}{l}3^{\$} \\
(42.9)\end{array}$ & 1 & 14.3 & 14.3 & 22 & 17 & $\begin{array}{l}8 \\
(36.3)\end{array}$ & 4 & 1 \\
\hline $\begin{array}{l}70- \\
79\end{array}$ & 15 & 15 & $\begin{array}{l}12 \\
(80.0)\end{array}$ & 6 & 40.0 & 40.0 & 10 & 10 & $\begin{array}{l}8 \\
(80.0)\end{array}$ & 5 & 50.0 & 50.0 & 19 & 17 & $\begin{array}{l}14 \\
(73.7)\end{array}$ & 5 & 2 \\
\hline $\begin{array}{l}80- \\
89\end{array}$ & 5 & 5 & $\begin{array}{l}5 \\
(100.0)\end{array}$ & 2 & 40.0 & 40.0 & 3 & 3 & $\begin{array}{l}3 \\
(100.0)\end{array}$ & 2 & 66.7 & 66.7 & 13 & 13 & $\begin{array}{l}12 \\
(92.3)\end{array}$ & 7 & 5 \\
\hline $90+$ & - & - & - & - & - & - & - & - & - & - & - & - & 1 & 1 & $\begin{array}{l}1 \\
(100.0)\end{array}$ & 0 & 0 \\
\hline Total & 158 & 140 & $\begin{array}{l}65 \\
(41.1)\end{array}$ & 25 & 15.8 & 17.9 & 92 & 84 & $\begin{array}{l}38 \\
(43.7)\end{array}$ & 16 & 18.4 & 19.0 & 92 & 79 & $\begin{array}{l}57 \\
(62.0)\end{array}$ & 25 & 2 \\
\hline
\end{tabular}

Age-stratified hospitalisation rates, CFR and IFR are presented and compared to UK national statistics for the general population. UK national data is sourced Care statistics between $1 / 1 / 2020$ and $29 / 3 / 2021$; age not provided for one patient with CVID who died, $\$$ excludes one patient admitted with immune thromb SARS-CoV-2 infection, * excludes 2 patients attending emergency department but not admitted to hospital.

Univariate analysis demonstrated that increasing age, lower baseline lymphocyte count, receipt of prophylactic antibiotics, and the presence of chronic lung disease, cardiovascular disease, chronic liver disease, diabetes mellitus and chronic gastrointestinal disease were all associated with a significantly increased risk of mortality from COVID-19 in these individuals (Fig. 1, Supplementary Table 1). 64.0\% of individuals who died had a pre-COVID lymphocyte count less than $1.0 \times 10^{9} / \mathrm{L}$ compared to $21.8 \%$ who survived $(\mathrm{p}<0.0001)$. In multivariate analysis, a low pre-SARS-CoV-2 infection lymphocyte count and receipt of prophylactic antibiotics were independently associated with an increased risk of mortality from COVID-19 in these individuals (Supplementary Table S2). Six patients had serological responses to natural infection measured; $100 \%$ were found to be positive ( 5 spike glycoprotein antibody positive, 1 nucleocapsid antibody positive).

\section{PID receiving immunoglobulin replacement therapy}

One-hundred and fifty-nine cases of SARS-CoV-2 infection in individuals with PID receiving IgRT have been recorded in this case series. The median age of these individuals was 40 years (IQR 28.0-56.3), 44.7\% ( $n=71 / 159)$ were female and in addition to IgRT, 55.3\% $(n=88 / 159)$ were receiving prophylactic antibiotics. $41.5 \%(n=66 / 159)$ were hospitalised as a result of SARS-CoV-2 infection, a further 2 patients attended the emergency department but were not admitted to hospital and 1 patient was admitted for a flare of immune thrombocytopenia purpura, temporally associated with SARS-CoV-2 infection. IFR in 
this subgroup was $16.3 \%(n=26 / 159)$ and an increased CFR were observed in all age brackets above 20-29 compared to UK national statistics (Table 2). Median age of death from COVID-19 in this cohort was 57.0 years compared to the UK national average of 83.0 years.

In univariate analysis, patients with PID receiving IgRT who died of COVID-19 were significantly older, had lower pre-SARS-CoV-2 infection lymphocyte counts (Fig. 1A) and had a significantly higher prevalence of chronic lung disease (specifically bronchiectasis), cardiovascular disease, chronic liver disease and diabetes mellitus than those who survived (Table 3 ). $61.5 \%$ of individuals who died had a pre-infection lymphocyte count of less than $1.0 \times 10^{9} / \mathrm{L}$ compared to $21.8 \%$ who survived $(\mathrm{p}<0.0001)$. In multivariate analysis, a higher pre-infection lymphocyte count was independently associated with survival but chronic liver disease and diabetes mellitus were independently associated with mortality (Table 4). 
Table 3

Univariate analysis of risk factors associated with mortality in PID with antibody deficiency. Median and interquartile ranges are provided for continuous variables. Differences between the distributions evaluated using 2-tailed Mann-Whitney U test. Differences between categorical variables are evaluated using 2-tailed Fisher exact test with ORs calculated using the

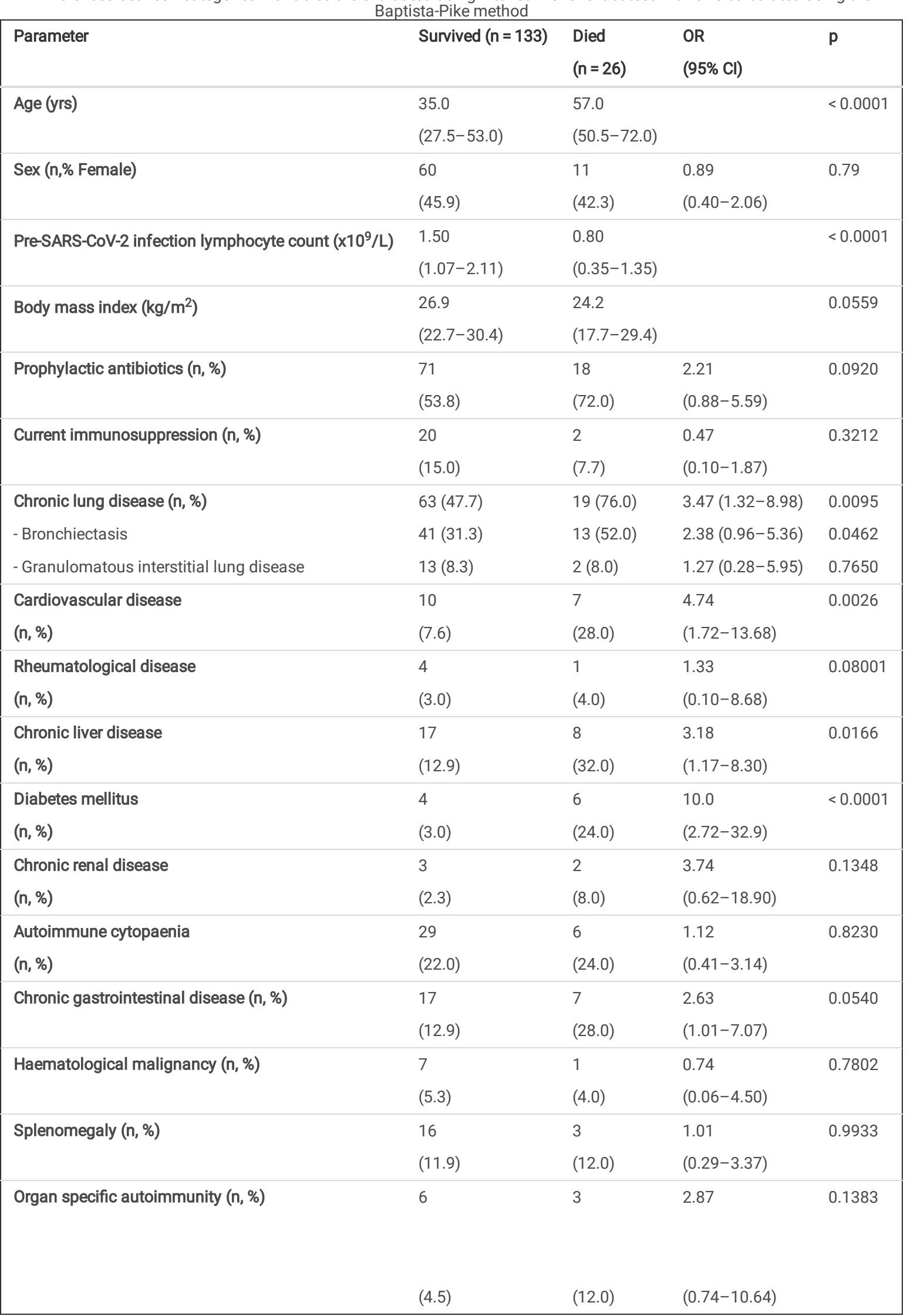


Table 4

Multivariate analysis of risk factors associated with mortality in PID with antibody deficiency

\begin{tabular}{|c|c|c|c|c|c|}
\hline Variable & Odds ratio & $95 \%$ confidence interval & $\mathbf{Z}$ & $P$ value & $P$ value summary \\
\hline Age & 0.97 & 0.93 to 1.01 & 1.36 & 0.17 & ns \\
\hline Male Sex & 2.61 & 0.58 to 13.2 & 1.23 & 0.22 & ns \\
\hline Pre SARS-CoV-2 infection lymphocyte count & 10.8 & 2.85 to 65.2 & 3.05 & 0.002 & ** \\
\hline Prophylactic antibiotics & 2.68 & 0.59 to 14.8 & 1.23 & 0.22 & ns \\
\hline Immune suppression & 0.18 & 0.009 to 1.55 & 1.4 & 0.16 & ns \\
\hline Bronchiectasis & 2.54 & 0.54 to 12.9 & 1.17 & 0.24 & ns \\
\hline GL-ILD & 0.54 & 0.02 to 6.12 & 0.45 & 0.65 & ns \\
\hline Cardiovascular disease & 6.76 & 0.64 to 88.8 & 1.55 & 0.12 & ns \\
\hline Rheumatological disease & 3.46 & 0.02 to 987 & 0.47 & 0.64 & ns \\
\hline Chronic liver disease & 16.5 & 2.05 to 160 & 2.56 & 0.01 & * \\
\hline Diabetes mellitus & 20.6 & 1.64 to 465 & 2.13 & 0.03 & * \\
\hline Chronic kidney disease & 1.81 & 0.02 to 64.5 & 0.29 & 0.77 & ns \\
\hline Autoimmune cytopenias & 0.07 & 0.004 to 0.61 & 2.18 & 0.03 & * \\
\hline Chronic gastrointestinal disease & 1.79 & 0.33 to 8.95 & 0.70 & 0.48 & ns \\
\hline Haematological malignancy & 0.64 & 0.001 to 27.8 & 0.17 & 0.87 & ns \\
\hline Splenomegaly & 0.37 & 0.02 to 5.08 & 0.72 & 0.47 & ns \\
\hline Other organ specific autoimmunity & 5.84 & 0.39 to 80.9 & 1.31 & 0.19 & ns \\
\hline
\end{tabular}

\section{X-linked agammaglobulinaemia}

Twenty-six patients with X-linked agammaglobulianemia were included (Table 1). The cohort of XLA patients were, on average, younger than other individuals with antibody deficiency (median age 29.5 years) and IFR was lower at $7.7 \%(n=2 / 26)$. Of the two individuals who succumbed to COVID-19, one had received lung transplantation for respiratory complications of their immunodeficiency [11]; the other was reported to suffer from nodular regenerative hyperplasia but had no pre-existing structural lung disease and died despite receiving treatment with dexamethasone, remdesivir, tocilizumab and convalescent plasma.

\section{Secondary immunodeficiency}

Ninety-two patients with SID, under the care of a clinical immunologist were included in this case series (Table 1). This cohort was, on average, older than the PID cohort with a median age of 63.0 years and outcomes were worse, with an IFR of $27.2 \%(n=25 / 92)$. Compared to UK national statistics, higher IFR were observed in all age brackets above 40 years (Table 2). Haematological malignancy and its treatment was the most common cause of secondary immunodeficiency in this cohort accounting for $56.5 \%(n=52 / 92)$ of cases: non-Hodgkin's lymphoma $(n=27)$, chronic lymphocytic leukaemia $(n=11)$ and plasma cell dyscrasias were the most common underlying diseases in this group. $55.8 \%(n=29 / 52)$ of these patients were hospitalised with and IFR of $23.1 \%$ $(n=12 / 52)$.

Individuals whose secondary immunodeficiency arose from underlying rheumatological disease were younger, more likely to be receiving immune suppression at the time of infection and had a higher IFR of $38.1 \%(n=8 / 21)$. In univariate analysis, patients with SID who died from COVID-19, had significantly lower preinfection lymphocyte counts than those who survived (Fig. 1, Table 5). 52.6\% of individuals who died had pre-SARS-CoV-2 lymphocyte count less than $1.0 \times 10^{9} / \mathrm{L}$ compared to $26.6 \%$ who survived $(p=0.03$ ). We also observed that in the SID cohort, patients receiving daily prednisolone as immunosuppression were far more likely to die than those not receiving daily prednisolone (mortality $44.0 \%$ vs. $7.46 \%, p<0.0001$ ). However, multivariate analysis did not show a significant independent effect of any of these variables (Supplementary Fig. 3). Seroconversion following natural infection was lower in SID than in CVID; 14 patients were assessed and $42.8 \%(n=6 / 14)$ were found to be seropositive following their illness compared to $100 \%(n=6 / 6)$ in CVID. 
Table 5

Univariate analysis of risk factors associated with mortality in SID

\begin{tabular}{|c|c|c|c|c|}
\hline Parameter & Survived $(n=67)$ & Died $(n=25)$ & OR $(95 \% \mathrm{Cl})$ & $\mathbf{p}$ \\
\hline Age (yrs) & $63.0(50.0-71.0)$ & $66.0(50.0-80.0)$ & & 0.21 \\
\hline \multirow[t]{2}{*}{ Sex (n,\% Female) } & $38(56.7 \%)$ & $15(60.0 \%)$ & 1.15 & 0.99 \\
\hline & & & $(0.47-2.91)$ & \\
\hline \multirow[t]{2}{*}{ Pre SARS-CoV-2 infection lymphocyte count (x109/L) } & 1.40 & 0.89 & & 0.03 \\
\hline & $(0.87-1.90)$ & $(0.60-1.20)$ & & \\
\hline \multirow[t]{2}{*}{ Body mass index $\left(\mathrm{kg} / \mathrm{m}^{2}\right)$} & 26.0 & 25.8 & & 0.67 \\
\hline & $(23.4-29.2)$ & $(21.3-32.6)$ & & \\
\hline \multirow[t]{2}{*}{ Immunoglobulin replacement (n, \%) } & 43 & 19 & 1.77 & 0.28 \\
\hline & $(64.2)$ & $(76.0)$ & $(0.64-5.08)$ & \\
\hline \multirow[t]{2}{*}{ Prophylactic antibiotics (n, \%) } & 40 & 15 & 1.01 & 1.01 \\
\hline & $(59.7)$ & $(60.0)$ & $(0.41-2.57)$ & \\
\hline \multirow[t]{2}{*}{ Current immunosuppression (n, \%) } & 26 & 14 & 2.01 & 0.14 \\
\hline & $(38.8)$ & $(56.0)$ & $(0.82-5.23)$ & \\
\hline Chronic lung disease (n, \%) & $23(34.4)$ & $12(48.0)$ & $1.77(0.70-4.44)$ & 0.23 \\
\hline - Bronchiectasis & $12(17.9)$ & $3(12.0)$ & $0.63(0.18-2.38)$ & 0.49 \\
\hline - Granulomatous interstitial lung disease & $\mathrm{n} / \mathrm{a}$ & $\mathrm{n} / \mathrm{a}$ & $\mathrm{n} / \mathrm{a}$ & $\mathrm{n} / \mathrm{a}$ \\
\hline \multirow[t]{2}{*}{ Cardiovascular disease (n, \%) } & 17 & 11 & 1.73 & 0.08 \\
\hline & $(25.4)$ & $(44.0)$ & $(0.16-1.19)$ & \\
\hline \multirow[t]{2}{*}{ Rheumatological disease (n, \%) } & 14 & 8 & 1.78 & 0.27 \\
\hline & $(20.9)$ & $(32.0)$ & $(0.61-5.18)$ & \\
\hline \multirow[t]{2}{*}{ Chronic liver disease (n, \%) } & 1 & 1 & 2.75 & 0.46 \\
\hline & $(1.49)$ & $(4.0)$ & $(0.14-52.9)$ & \\
\hline \multirow[t]{2}{*}{ Diabetes mellitus (n, \%) } & 7 & 5 & 2.14 & 0.23 \\
\hline & $(10.5)$ & $(20.0)$ & $(0.66-7.33)$ & \\
\hline \multirow[t]{2}{*}{ Chronic renal disease $(\mathrm{n}, \%)$} & 11 & 3 & 0.69 & 0.60 \\
\hline & $(16.4)$ & $(14.3)$ & $(0.19-2.74)$ & \\
\hline \multirow[t]{2}{*}{ Autoimmune cytopaenia (n, \%) } & 2 & 3 & 4.43 & 0.09 \\
\hline & $(2.99)$ & $(12.0)$ & $(0.84-25.72)$ & \\
\hline \multirow[t]{2}{*}{ Chronic gastrointestinal disease (n, \%) } & 5 & 1 & 0.52 & 0.55 \\
\hline & (7.5) & $(4.0)$ & $(0.04-4.24)$ & \\
\hline \multirow[t]{2}{*}{ Haematological malignancy (n, \%) } & 40 & 13 & 0.73 & 0.50 \\
\hline & $(59.7)$ & $(52.0)$ & $(0.30-1.83)$ & \\
\hline \multirow[t]{2}{*}{ Splenomegaly (n, \%) } & 3 & 0 & 0.00 & 0.28 \\
\hline & $(4.5)$ & $(0.0)$ & $(0.00-3.09)$ & \\
\hline \multirow[t]{2}{*}{ Organ specific autoimmunity (n, \%) } & 2 & 2 & 2.83 & 0.29 \\
\hline & $(2.99)$ & $(8.0)$ & $(0.42-18.59)$ & \\
\hline
\end{tabular}

\section{C1 inhibitor deficiency}

Data on the outcomes of 13 patients with $\mathrm{C} 1$ inhibitor deficiency were submitted, all of whom survived (Table 1); the median age of patients with $\mathrm{C} 1$ inhibitor deficiency was 45 years (IQR: 29.5-51.5) and $46.1 \%$ were female. Only one patient, a man in his 20 s with other pre-existing comorbidities, required hospital treatment specifically for COVID-19; another required hospitalisation for renal disease. Of note, additional information was submitted for 2 patients implicating SARS-CoV-2 infection as a precipitating factor for concurrent flares of hereditary angioedema, as has been previously reported [12, 13]. 


\section{Acute treatments for COVID-19}

144 patients in this cohort required hospital treatment for COVID-19, with an overall inpatient survival of $61.8 \%(n=89 / 144)$. 22.9\% of hospitalised patients ( $n$ $=33 / 144$ ) received a targeted COVID-19 treatment within the RECOVERY trial, on a compassionate use basis, or as standard of care following adoption of treatment proven to be efficacious in the RECOVERY trial (Table 6). In this cohort, 20 patients received dexamethasone, 26 patients received remdesivir and 10 patients received anti-SARS-CoV-2 antibody-based therapies (e.g. REGEN-COV2, convalescent plasma) either as a monotherapy or in combination: the survival rates of individual patients treated with dexamethasone and/or remdesivir and/or antibody-based treatments exceeded $75 \%$. Five patients received tocilizumab as a monotherapy or in combination: survival rates in this cohort were $20 \%$.

Table 6

Targeted COVID-19 treatments used in patients with PID or SID

\begin{tabular}{|c|c|c|c|}
\hline Treatment & $\mathrm{n}$ & Age (median) & $\begin{array}{l}\text { Survived } \\
(\mathrm{n}, \%)\end{array}$ \\
\hline Dexamethasone overall & 20 & 51.3 & $15(75.0)$ \\
\hline - Dexamethasone monotherapy & 5 & 46.0 & $4(80.0)$ \\
\hline - Dexamethasone + remdesivir & 8 & 56.0 & $8(100.0)$ \\
\hline Remdesivir overall & 26 & 54.5 & $22(84.6)$ \\
\hline - Remdesivir monotherapy & 7 & 57.0 & $7(100.0)$ \\
\hline Antibody based therapies overall & 10 & 52.5 & $8(80.0)$ \\
\hline - REGEN-COV-2 monotherapy & 1 & 70 s & $1(100.0)$ \\
\hline - REGEN-COV2 + remdesivir & 1 & $80 s$ & $1(100.0)$ \\
\hline - REGEN-COV2 + remdesivir + dexamethasone & 2 & 33.0 & $2(100.0)$ \\
\hline - Convalescent plasma + remdesivir & 4 & 53.5 & $3(75.0)$ \\
\hline Tocilizumab overall & 5 & 60.0 & $1(20.0)$ \\
\hline - Tocilizumab monotherapy & 1 & $60 \mathrm{~s}$ & $0(0.0)$ \\
\hline - Tocilizumab + dexamethasone & 1 & 70 s & $0(0.0)$ \\
\hline - Tocilizumab + dexamethasone + remdesivir & 1 & 50 s & $0(0.0)$ \\
\hline - Tocilizumab + dexamethansone + remdesivir + convalescent plasma & 1 & $40 s$ & $0(0.0)$ \\
\hline - Tocilizumab + dexamethansone + remdesivir + REGEN-COV2 & 1 & 70 s & $1(100.0)$ \\
\hline
\end{tabular}

\section{Discussion}

The United Kingdom has recorded amongst the highest number of cases (8,918 per 100,000 population) and deaths (193.9 per 100,000 population) from COVID-19 in the world [14]. Despite public health measures to minimise the exposure of clinically extremely vulnerable individuals to SARS-CoV-2 [15], the pandemic has disproportionately affected patients with PID and SID. In this large nationwide study, we demonstrate an increased risk of mortality from COVID19 in individuals with PID requiring antibody replacement therapy and individuals with CVID in all age groups above 20 years old compared to the UK general population. In patients with SID, an increased risk of mortality emerges beyond 40 years of age. Inpatient mortality in patients with immunodeficiency was higher than the general population (38.2\% vs $26.0 \%$ ) [1]. In comparison to the general population, where the median age of death from COVID-19 was 83.0 years, immunodeficiency patients died, on average, up to 26 years younger than the general population.

The risk factors for poor outcome from COVID-19 in patients with primary immunodeficiencies closely mirror those in the general population [16]; increasing age and common comorbidities were all associated with an increased risk of death in univariate analysis; chronic liver disease and diabetes mellitus (PID on $\operatorname{lgRT}$ ) and receipt of prophylactic antibiotics (CVID) were independently associated with death in multivariate analysis of these cohorts. In SID, no additional independent risk factors were observed, but the overall CFR of $31.6 \%$ compared to $2.95 \%$ in the UK general population illustrates the vulnerability of individuals requiring long-term immunological support following treatment for other diseases. Furthermore, the CFR of $38.1 \%$ for patients with rheumatological disease in this study is markedly higher than the estimated CFR of $5.6 \%$ for unselected rheumatoid arthritis patients demonstrating the subgroup of patients with SID are at especially high risk of poor outcome [17]. Our data also highlights that those individuals with secondary immunodeficiency who continue to receive treatment with any daily dose of prednisolone also appear at increased risk of mortality. Reassuringly and concordant with other UK studies in immunocompromised children [7], we found no increased risk of mortality amongst in the 18 paediatric patients within this study, although $27.7 \%$ were hospitalised. 
One striking observation from this study is that pre-existing lymphopenia is independently associated with mortality in all three groups with immunodeficiency. Previous studies have demonstrated that pre-existing lymphopenia is independently associated with an increased risk of developing pneumonia, skin infections, urinary tract infections, sepsis and endocarditis in the Danish general population [18] and an increased risk of mortality from pneumonia in the UK general population [19]. Peripheral CD4 and CD8 T cell lymphopenia and dysregulated T cell responses have also been associated with severe disease during acute COVID-19, although some studies suggest this observation is secondary to lymphocyte redistribution during acute illness [20,21]. Characterising the nature of pre-existing lymphopenia and its impact on functional immunity with respect to outcomes from infectious disease, is an important research priority in both the general population and patients with immune deficiency.

There is a paucity of evidence regarding the efficacy of treatments for COVID-19 in patients with PID and SID [22]. In this case series, compared to an overall inpatient survival following COVID-19 of $61.8 \%$, survival was improved in individuals who received dexamethasone (75.0\%), remdesivir (84.6\%) or antibodybased treatments $(80.0 \%)$ as a monotherapy or in any combination. In contrast, only $20 \%$ of individuals receiving tocilizumab survived. These data require cautious interpretation; specific data on the timing of these pharmacological interventions in relation to disease onset and COVID-19 severity was not gathered as part of this study and the number of treated individuals is small. However, it is possible, or even likely, that modulation of the immune response in a patient with immunodeficiency is different to the general population. For example, although convalescent plasma demonstrated no benefit in healthy individuals during acute severe COVID-19 infection [23], antibody-based treatments appear effective herein and in case reports of antibody deficiency patients where ex vivo studies have confirmed viral neutralization [24]. Furthermore, polyclonal immunoglobulin replacement is the standard of care in the prevention of chronic sinopulmonary infection in individuals with antibody deficiency and hyperimmune serum may be used for post-exposure prophylaxis in seronegative individuals against certain infectious diseases, a concept supported by early data in anti-SARS-CoV-2 monoclonal antibody trials [25, 26]. Similarly, inhibition of the IL- 6 axis in individuals already suffering from an existing immunodeficiency, may have unintended consequences despite success in randomised control trials in previously healthy individuals [27].

The major strength of this study is its size and comprehensive representation amongst UK PIN affiliated centres across the United Kingdom reducing the potential for bias seen in smaller case series and facilitating the enrolment of large numbers of patients with rare disease. The work confirms the increased risk of mortality from COVID-19 we observed in our original case series [5] and builds upon it by providing revised estimates of the magnitude of that risk in relation to the UK general population and the determinants of that risk in clinically relevant disease subgroups. However, this study remains a clinicianreported registry and we are unable to guarantee that all SARS-CoV-2 infections in patients with PID or SID have been captured by this study. Furthermore, bias may exist within the SID cohort described herein; by definition, these individuals have been referred to a Clinical Immunologist for immunological assessment which may enrich for more severe phenotypes of SID. Cases of SARS-CoV-2 in paediatric patients remain underrepresented by this study, but similar outcomes in a larger cohort of UK patients have been reported elsewhere [7]. Owing to the retrospective nature of this study, we are unable to characterize the nature or longevity of serological responses of individuals with PID or SID following natural infection; however, the COV-AD study, a national UK study studying the cellular and humoral response to SARS-CoV-2 natural infection and vaccination is in progress and will be able to inform upon these important immunological questions.

In summary, our study highlights the burden of morbidity and mortality in individuals with PID and SID following infection with SARS-CoV-2 and elucidates independent risk factors associated with poor outcome. The impact of the COVID-19 on individuals with PID and SID cannot be underestimated; these data must inform public health policy, including the urgent provision of anti-SARS-CoV-2 antibody-based therapies this population, to minimise the risk of poor outcome during future waves of the evolving pandemic.

\section{Abbreviations}




\begin{tabular}{|ll|}
\hline AID & Activation induced cytidine deaminase \\
\hline ALPS & Autoimmune lymphoproliferative syndrome \\
\hline AR & Autosomal recessive \\
\hline CARD 2 & BTB Domain And CNC Homolog 2 \\
\hline CFR & Caspase Recruitment Domain Family Member 11 \\
\hline CGD & Chronic granulomatous disease \\
\hline CID & Combined immunodeficiency \\
\hline CTLA-4 & Cytotoxic T-lymphocyte associated protein 4 \\
\hline CVID & Common variable immunodeficiency disorder \\
\hline GATA-2 & GATA-binding factor 2 \\
\hline GoF & Gain of function \\
\hline ICOS & Inducible T cell costimulator \\
\hline IFR & Infection:fatality ratio \\
\hline MBL & Mannose binding lectin \\
\hline NFKB & Nuclear factor kappa B \\
\hline PID & Primary immunodeficiency \\
\hline SID & Secondary immunodeficiency \\
\hline SPAD & Specific polysaccharide antibody deficiency \\
\hline STAT & Signal transducer and activator of transcription \\
\hline TCC & Terminal complement complex \\
\hline TPP-2 & Tripeptidyl Peptidase 2 \\
\hline XL & X-linked \\
\hline XLA & X-linked agammaglobulinaemia \\
\hline XLP & X-linked lymphoproliferative disorder \\
\hline
\end{tabular}

\section{Declarations}

\section{Acknowledgements}

The authors would like to acknowledge the wider immunology community, in particular those individuals living with primary and secondary immunodeficiency and their families for their patience and resilience throughout the COVID-19 pandemic.

\section{Funding}

No specific funding was received for this study.

Competing interests:

The authors declare no competing interests

\section{Availability of data and materials}

All available data is presented within the figures and tables of this manuscript.

\section{Code availability}

No code has been used in the preparation of this manuscript.

\section{Author Contributions}

AMS, SOB, SS and AGR conceived the study. AMS collated the data, analyzed the data, produced the first draft of the manuscript and revised the manuscript. SOB, SS and AGR are the joint senior authors of this manuscript. All authors contributed to data collection and reviewed the revised manuscript prior to submission. 


\section{Ethics approval}

This study reports fully anonymized, routinely collected data regarding patient outcome. No specific ethical approval is required for collation or publication in accordance with HRA guidance.

\section{Consent to participate}

Not applicable

\section{Consent for publication}

Not applicable

\section{References}

1. Docherty AB, et al., Features of 20133 UK patients in hospital with covid-19 using the ISARIC WHO Clinical Characterisation Protocol: prospective observational cohort study. Bmj, 2020. 369: p. m1985.

2. Bennett KE, et al. Underlying conditions and risk of hospitalisation, ICU admission and mortality among those with COVID-19 in Ireland: A national surveillance study. Lancet Reg Health Eur. 2021;5:100097.

3. Meyts I, et al., Coronavirus Disease 2019 in patients with inborn errors of immunity: an international study. Journal of Allergy and Clinical Immunology.

4. Marcus N, et al. Minor Clinical Impact of COVID-19 Pandemic on Patients With Primary Immunodeficiency in Israel. Front Immunol. 2020;11:614086.

5. Shields AM, et al., COVID-19 in patients with primary and secondary immunodeficiency: The United Kingdom experience. J Allergy Clin Immunol, 2020.

6. Al Yazidi LS, et al. COVID-19 AND PRIMARY IMMUNODEFICIENCY: ONE-YEAR EXPERIENCE. J Paediatr Child Health. 2021;57(4):594.

7. Chappell HP, Driessens R, Tarr C, Irving A, Tighe W, Jackson P, Harvey-Cowlishaw H, Mills T, Shaunak L, Gbesemete S, Leahy G, Lucas A, Faust J, de Graaf S, H.; ImmunoCOVID19 Study Group, Immunocompromised Children and Young People Are at No Increased Risk of Severe COVID-19. Preprint, 2021. https://papers.ssm.com/sol3/papers.cfm?abstract_id=3893560.

8. Delavari S, et al. Impact of SARS-CoV-2 Pandemic on Patients with Primary Immunodeficiency. J Clin Immunol. 2021;41(2):345-55.

9. Goudouris ES, et al., Outcome of SARS-CoV-2 Infection in 121 Patients with Inborn Errors of Immunity: A Cross-Sectional Study. J Clin Immunol, 2021: p. $1-11$.

10. Buckland MS, et al. Treatment of COVID-19 with remdesivir in the absence of humoral immunity: a case report. Nat Commun. 2020;11(1):6385.

11. Loh SY, et al., Possible COVID-19 reinfection in a patient with X-linked agammaglobulinaemia. BMJ Case Rep, 2021. 14(3).

12. Belbézier A, et al. COVID-19 as a trigger of acute attacks in people with hereditary angioedema. Clin Exp Allergy. 2021;51(7):947-50.

13. Grumach AS, et al. COVID-19 affecting hereditary angioedema patients with and without C1 inhibitor deficiency. J Allergy Clin Immunol Pract. 2021;9(1):508-10.

14. Government UK. Coronavirus (COVID-19) in the UK, Public Health England; https://coronavirus.data.gov.uk/details/deaths.

15. Government UK. Guidance on protecting people who are clinically extremely vulnerable from COVID-19, Public Health England: https://www.gov.uk/government/publications/guidance-on-shielding-and-protecting-extremely-vulnerable-persons-from-covid-19/guidance-on-shieldingand-protecting-extremely-vulnerable-persons-from-covid-19.

16. Williamson EJ, et al., OpenSAFELY: factors associated with COVID-19 death in 17 million patients. Nature, 2020.

17. England BR, et al. Risk of COVID-19 in Rheumatoid Arthritis: A National Veterans Affairs Matched Cohort Study in At-Risk Individuals. Arthritis Rheumatol; 2021.

18. Warny M, et al. Lymphopenia and risk of infection and infection-related death in 98,344 individuals from a prospective Danish population-based study. PLoS Med. 2018;15(11):e1002685.

19. Hamilton F, Arnold D, Payne R. Association of prior lymphopenia with mortality in pneumonia: a cohort study in UK primary care. Br J Gen Pract. 2021;71(703):e148-56.

20. Vabret N, et al. Immunology of COVID-19: Current State of the Science. Immunity. 2020;52(6):910-41.

21. Adamo S, et al., Profound dysregulation of T cell homeostasis and function in patients with severe COVID-19. Allergy, 2021.

22. Moran E, et al. Persistent SARS-CoV-2 infection: the urgent need for access to treatment and trials. Lancet Infect Dis; 2021.

23. RECOVERY collaborative group. Convalescent plasma in patients admitted to hospital with COVID-19 (RECOVERY): a randomised controlled, open-label, platform trial. Lancet. 2021;397(10289):2049-59.

24. McKemey E, et al. Resolution of Persistent COVID-19 After Convalescent Plasma in a Patient with B Cell Aplasia. J Clin Immunol. 2021;41(5):926-9.

25. Chen P, et al. SARS-CoV-2 Neutralizing Antibody LY-CoV555 in Outpatients with Covid-19. N Engl J Med. 2021;384(3):229-37.

26. Weinreich DM, et al. REGN-COV2, a Neutralizing Antibody Cocktail, in Outpatients with Covid-19. N Engl J Med. 2021;384(3):238-51.

27. RECOVERY collaborative group. Tocilizumab in patients admitted to hospital with COVID-19 (RECOVERY): a randomised, controlled, open-label, platform trial. Lancet. 2021;397(10285):1637-45.

\section{Figures}


Figure 1

A) PID on immunoglobulin replacement $(n=159)$
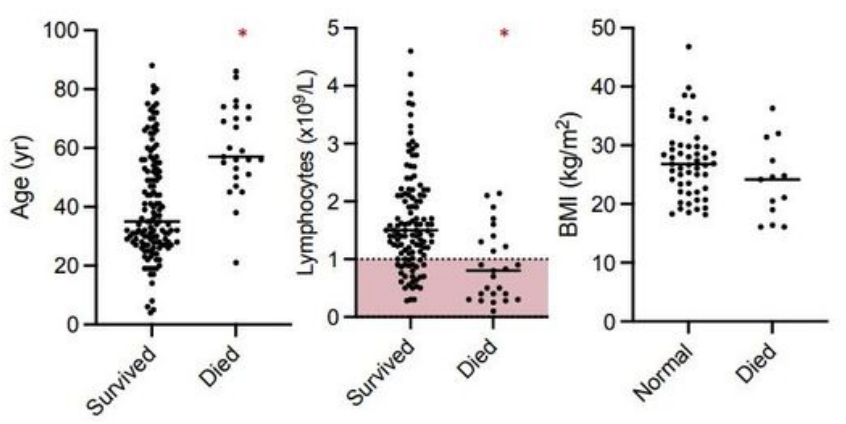

C) Symptomatic secondary immunodeficiency $(n=92)$
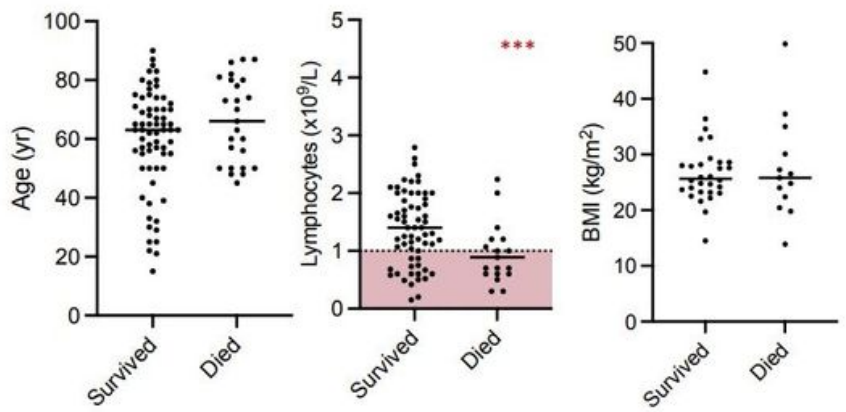

B) CVID phenotype $(n=93)$

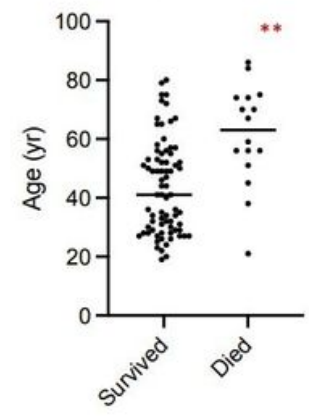

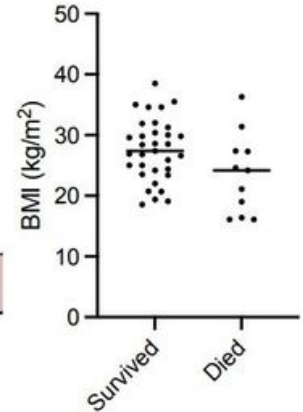

Figure 1

Risk factors for mortality from COVID-19 in patients with PID and SID Age, baseline lymphocyte count and body mass index are compared between COVID-19 survivors and non-survivors in patients with (A) PID on immunoglobulin replacement, (B) Common variable immunodeficiency and (C) Secondary immunodeficiency. Statistical comparison of the groups is made using the two-tailed Mann-Whitney U test. * $p<0.0001,{ }^{\star *} p=0.003,{ }^{* \star *} p=0.03$. Red shaded area denotes lymphopenia $(<1.0 \times 109 / \mathrm{L})$.

\section{Supplementary Files}

This is a list of supplementary files associated with this preprint. Click to download.

- SupplementaryTablesFINAL.docx 